S M Yasir Arafat ${ }^{1,2 *}$, Konstantinos Papadopoulos ${ }^{3}$, Mohammad S I Mullick4, Md. Saleh Uddin ${ }^{4}$

\title{
The Vulnerability and Resiliency of Childhood
}

\author{
'Department of Psychiatry, CARe Medical College, Dhaka, Bangladesh. \\ ²Biomedical Research Foundation, Dhaka, Bangladesh \\ ${ }^{3}$ Middlesex University, UK. \\ 4Department of Psychiatry, Bangabandhu Sheikh Mujib Medical University, Bangladesh \\ *email: arafatdmc62agmail.com
}

DOI: 10.2478/gp-2019-0010

Received: 26 April 2019; Accepted: 12 May 2019.

\begin{abstract}
Life is full of stressors, which have to be confronted efficiently to grow up. However, reaction to stressors is personalized, complex and coordinated. Vulnerable persons adjust poorly to stressors and express inappropriate responses, while resilient persons practice adaptive physiological and psychological responses. Promotion of resiliency is an intricated issue, which demands strategies at both macro and micro-level. Microlevel strategies are focused on the community, family and individual level, while macrolevel strategies formulate the principles. Nevertheless, prediction of vulnerability and resiliency is really a challenge, as different persons facing same stressors react differently. Some are growing as resilient and others as vulnerable. We aimed to discuss resiliency, vulnerability, importance in relation to health outcome, promotion of resiliency and controversies of vulnerability and resiliency.
\end{abstract}

\section{Keywords}

Childhood vulnerability, childhood resiliency, chronic stress, vulnerable factors, resilient factors

\section{INTRODUCTION}

Stress bears different meaning for different people, who experience it in different conditions (Fink, 2009). There are many different definitions of stress; among the first ones was the one given by the 'father of stress' (Szabo et al., 2012). Hans Selye stated that, 'Stress is the nonspecific response of the body to any demand' (Selye, 1976). The American Psychological Association has divided stress into 3 categories according to the characteristic symptoms, duration and treatment approaches. These categories are acute, episodic acute and chronic (Miller \& Smith 1993). Acute and episodic acute stress can be eustress in small doses and are the most common forms of stress (Hammen et al., 2009).

Unlike the first two categories, chronic stress is not exciting but can destroy bodies, minds and lives; can affect people of all ages and genders and can lead not only to physical but also to psychological health problems (Anderson, 1998). Chronic stress can be defined as the response to emotional pressure experienced for a prolonged period of time, when the individuals have the feeling of having little or no control (Carlson, 2013). Hans Selye was the first to study chronic stress in lab mice and his experiments led to the recognition of the 'general adaptation syndrome'. The theory concluded that humans exposed to prolonged stress could experience hormonal breakdown, and subsequently, develop conditions such as heart disease and elevated blood pressure (Selye, 1952).

Most vulnerable to chronic stress appear to be children and adolescents. Types of chronic stressors that children and youth experience include frequent parental arguments, divorce, chronic illness (parental cancer), neighbourhood crime, caregiving for a parent or sibling, and trying to adapt to another culture (Terzian et al., 2010). The prevalence of children suffering from a chronic illness varies widely, but the overall rate is $10 \%$ to $20 \%$ (Janse et al., 2005). Children with chronic illnesses are more likely to have emotional, behavioural and psychiatric symptoms than healthy children (Knapp \& Harris, 1998) and may be psychologically affected or traumatized by medical treatments (Stuber et al., 2006). Children and adolescents appear to be particularly vulnerable when they have one of the following characteristics:

- low socioeconomic status,

- a tendency to blame negative events on themselves,

- lack of sufficient amounts of sleep, food, nutrition, or exercise, 
- prior history of psychological or behavioural disorder (such as attachment-related problems or anxiety), or a low tolerance for stress,

- lack of social support (from peers or parents),

- school circumstances and academic difficulties,

- multiple co-occurring stressors, for example, living in a family environment with high conflict or in a neighbourhood with high crime, or

- residence in a socially isolated neighbourhood.

(Thoresen \& Eagleston, 1983; Gerstoff et al., 2003)

Signs of chronic stress in children and youth can be either physical such as muscle tension headaches and loss of energy; emotional such as anxiety and nervousness; and behavioural such as weight loss/gain (Romer, 1993). Recently, researchers have shown that chronic stress generates long-term changes in the brain that may explain why people suffering from chronic stress are more prone to mental problems such as anxiety and mood disorders later in life (Chetty et al., 2014). Chronic stress in children and adolescents and coping with it is usually related to the adaptation capacity to illness. Recent studies support a control-based model of coping that includes efforts to act on the source of stress or one's emotions (primary control or active coping), efforts to adapt to the source of stress (secondary control or accommodative coping), and efforts to avoid or deny the stressor (disengagement or passive coping) (Compas et al., 2012).

Nobody wants to be to be an alcoholic, a psychoactive substance abuser, or HIV infected (Chandler et al., 2015). However, some people have these characteristics and are responsible for adverse childhood experiences, which may raise the perceived stress and initiate or precipitate risky health behaviours for some children (Chandler et al., 2015). Growing evidence revealed both short- and long-term adversities to health and well-being due to adverse life experiences in childhood, particularly when adversities are enduring, cumulative, or occurring during sensitive periods in early developing phase (Masten \& Barnes, 2018). Nevertheless, many people manage to fight back lots of adversities in their childhood. This phenomena has been explained as resiliency. Resiliency has been emerging since 1970 in research when researchers observed significant variability among groups of children struggling with multiple risks and adversities (Masten \& Barnes, 2018). In the subsequent half century, researchers in various disciplines, most notably in behavioural science, psychiatry, paediatrics, and education, have been trying to explain the observable variation (Masten \& Barnes, 2018).

\section{IMPORTANCE IN RELATION TO HEALTH OUTCOME}

Life is full of stressors which have to be confronted efficiently to stay healthy. Eustress, such as learning a new skill, is an essential component of growth and development of a child. The child increases motivation and resiliency from each positive stress response (Franke, 2014). However, every individual reacts differently to deal with similar stressors. Vulnerable persons poorly adjust to stressors and express inappropriate responses, while the resilient persons can distinguish the adversity as less stressful and practice adaptive physiological and psychological responses (Franklin et al., 2012). The variations in individual resilience/vulnerability have been noticed across age, gender and culture, which depends on complex interaction of genetic, environmental and personal experiences (Franklin et al., 2012; Franke, 2014). However, the exact underlying mechanisms are yet to be identified. Adaptive coping of stress is essential to minimize the unhealthy consequences as well as to ascertain the degree of resiliency and vulnerability, which has been influenced by the interaction of neuroendocrine and neuroimmunological systems in response to threats (Franklin et al., 2012). This interaction plays a vital role in determining whether stress-related disorders develop or not. For instance, passive stress coping strategy is associated with the development of depressive disorder and post-traumatic stress disorder (PTSD) (Franklin et al., 2012). Passive coping strategies includes denial, avoidance of conflicts, suppression of emotions, and behavioural disengagement, which are certainly maladaptive and provide short-term escape (Franklin et al., 2012; Wood \& Bhatnagar, 2015). On the other hand, active coping strategies involve the individual in optimism and positive secondary appraisal to deal with a challenge, face fears, participate in problem solving and seek social support. These can produce long-term resilience (Franklin et al., 2012; Wood \& Bhatnagar, 2015; Beutel et al., 2017). Active coping is associated with the development of resiliency and passive coping is associated with vulnerability (Wood \& Bhatnagar, 2015; Beutel et al., 2017).

Neuroendocrine changes happen in response to toxic stressors, which change the cortisol response abnormally resulting in dysregulation of immune system, persistent state of being inflamed, raises the chances of infections. Stressful life events are associated with depressive illness, behavioural dysregulation, PTSD and even with psychosis. Adults who encountered adverse life events in childhood experience more physical diseases and mental disorders in adulthood, maladjustment and poor health outcomes (Franklin et al., 2012; Franke, 2014; Wood \& Bhatnagar, 2015; Beutel et al., 2017). 
The poor health outcomes are varied and include alcoholism, chronic obstructive pulmonary disease, depressive disorders, malignancies, obesity, increased suicidal behaviour, ischemic heart disease and multiple other illnesses (Franke, 2014).

\section{RESILIENCY}

Resiliency concept has become more common recently, especially on developmental psychology contexts (Beutel et al., 2017; Hornor, 2017; Masten \& Gewirtz, 2006). It is a contextual as well as a dynamic concept (Broekman, 2011; Hornor, 2017). It refers to a dynamic equilibrium in which people demonstrate positive adaptation in spite of threats (Judge, 2005; Masten \& Gewirtz, 2006). It is the experience that has emerged from adversities with good outcomes (Daniel, 2010). Initially, it was thought as a person-centred matter; however, later it was revealed as complex interactions between an individual and his/ her surrounding ecology (Van Rensburg et al., 2015). Resilience is the capability of positive adaptations or the ability to maintain or regain mental health, despite experiencing adverse situations (Franke, 2014; Wood \& Bhatnagar, 2015; Moreno et al., 2016). Others defined it as 'an outcome in the face of adversity or as a process mediating the response to stress or trauma' (Beutel et al., 2017). It depends on an individual's multiple factors that change over time (Franke, 2014). Identified factors responsible for developing resiliency among child(ren) are higher IQ, easy temperament, good esteemed competence, a positive evaluation of self, a realistic sense of control of situation, empathy, social problem-solving skills, adequate social support, good marital relationship of parents, the physical and mental health of the parents, having support from adults and approach-oriented temperament; low neuroticism as personality trait, perceived care of the parents, good peer relationships and the parent's sense of efficacy (Franke, 2014; Beutel et al., 2017; Horner, 2017; Judge, 2005). Previous experience of adversity overcoming with adaptive behaviour reinforce the resiliency (Franke, 2014). Predicting factors of resiliency among children include a solid relationship between the child and parent, that is, secure attachment behaviour and high-quality teacher child relation (Franke, 2014; Beutel et al., 2017).

Previous researches revealed that the capability of an individual to respond positively to physiological, psychological or social threats in the surrounding environment as the most prominent factor of resiliency (Beutel et al., 2017). An enduring pattern of individual characteristics was identified, which is related with successful adaptation to the adverse situations in early life (Charney, 2003). The characteristics include higher intellectuality, adequate regulation of own emotions, good attachment, positive self-esteem, optimistic and altruistic attitude, an excellent capability to work as learned helpfulness instead of learned helplessness and active coping strategy to challenge the stressors (Charney, 2003). Protective factors can be categorized into three areas, that is, individual factors such as intelligence and temperament, childhood relationship quality such as attachment and environmental factors such as neighbourhood, school and overall social system of the country (Hornor, 2017; Arafat et al., 2018). Regardless the factors, stress is a fundamental issue to develop resilience intermingled with personal temperament and previous experiences. The situation can be exemplified by comparing the issue with the development process of our immunity where immunity develops after the infections (Hornor, 2017). Nowadays, in many situations, immunity can be produced by injecting pathogens without their virulence, which might be a challenge to find out similar mechanisms. However, systemic desensitization strategies for the treatment of phobia has been proved as an effective treatment (Hornor, 2017). But, using those strategies before the onset of illness is yet to be tested.

\section{VULNERABILITY}

Like resiliency, vulnerability is also thought as a probabilistic as well as a conditional construct (Masten \& Gewirtz, 2006). It is thought as the predisposition or susceptibility of an individual to specific disorder or maladaptive outcome in responses of challenges of threats (Masten \& Gewirtz, 2006). Raised sensitivity to stress with a compromised resource for social and psychological support to help with stress coping skills, inadequate social support, developmental delays, abusive parenting, and maladaptive behaviours in response to adversity were found to be the factors that make an individual more vulnerable (Franke, 2014).

Experiences of early period of childhood play a pivotal role in how the brain evolves and functions. Complex interactions of individual child and surrounding environment affect longterm learning, behaviour and health (Franke, 2014). Children with early life experience of toxic stress are at risk of developing and enduring adverse health problems, such as maladaptive coping skills, poor stress coping, unhealthy routines, mental disorders and physical illness, premature birth, childhood poverty (Evans \& Kim, 2013; Masten \& Gewirtz, 2006; Franke, 2014). These may not manifest until adulthood. Vulnerability factors include challenging social ecosystem such as violent living condition, adverse life events such as death of parents and biological factors such as genetic predispositions (Van Rensburg et al., 2015) 


\section{MODELS OF VULNERABILITY AND RESILIENCY}

\section{Diathesis-Stressor Model}

According to this popular model, a combination of two certain factors are needed to develop a condition. Thus, to develop a disorder, the individuals have to be predisposed in earlier life, and subsequently, have to come across stressful life events (Masten \& Gewirtz, 2006). A combination of these two factors are necessary. Those who are not predisposed, will not develop a disorder after facing stressors, while those who are predisposed, they will develop disorders. Surprisingly, accurate precision of predisposition and effect of stressor are still elusive. Different individual reacts differently with same stressors, as it is modified by the personal construct of that individual.

\section{Compensatory and Protective Factor Model}

This model encompasses factors those are typically unexplained by diathesis-stressor model (Masten \& Gewirtz, 2006). Availability of good influences (resource) may counterbalance the negative factors as well as reactions, reactive to the stressors (Masten \& Gewirtz, 2006). Strategies focusing on the reduction of vulnerability, along with a supplementation of adequate resources to cope with stressors, would act in an additive fashion to change the reactions in positive direction (Masten \& Gewirtz, 2006). Freely accessible information, adequate self-control, good anger management skills could increase the adaptability as a whole. Supportive parenting figures, ideal mentoring to the children who are vulnerable with poor social capital foster the positive development as well as buffer the frightening experiences after exposing the stressors (Masten \& Gewirtz, 2006). Parents play a demonstrative role in developing resiliency or vulnerability among children by acting as modifier in the developing period of life. Proper strategies targeting the modification of early life adversities should be prioritized as frontline, which can protect the children and/or modify the mediators (parents) (Masten \& Gewirtz, 2006).

\section{Adaptive Pathways and Dynamic Developmental Model}

Continuous equilibrium with the surrounding environment is mandatory to ensure a healthy life. Hence, dynamic adaptation of an individual is a silent but enduring mechanism to sustain in this everchanging world (Masten \& Gewirtz, 2006). Every stroke of life moves the life forward either in a negative or positive direction. Every stressor changes our ways and the direction of that changes are defined by the adaptation of that individual. Thus, focusing on the adaptations in positive ways helps a person to maintain bidirectional beneficial interactions (Masten \& Gewirtz, 2006).

\section{Epigenetics}

Epigenetics deal with modifiable changes in gene expression without changing the underlying DNA sequence (Hornor, 2017). It has an important role in developing of resiliency as well as vulnerabilities. Previous research revealed that, the serotonin transporter gene (SLC6A, 5-HTTLPR) polymorphism may have an effect (Hornor, 2017). Depression and other internalizing disorders are associated with short alleles and substance abuse. High sexual behaviours are associated with long alleles of 5-HTTLPR, and these may be associated with development of resiliency after exposing maltreatment and trauma in childhood (Hornor, 2017). Anti-social behaviours may be associated with the polymorphism of the MAOA gene among individuals who are exposed to childhood trauma and can raise the vulnerability (Hornor, 2017; Masten \& Gewirtz, 2006).

\section{PROMOTING RESILIENCE}

Resiliency promotion is an intricated issue, which demands strategies at both macro- and microlevel (Hornor, 2017). The microlevel strategies focus on the community, family and individual level, while macrolevel strategies formulate options (Hornor, 2017). Strategies of macro intervention demonstrates the economic policy, social dogma, community set up, and cultural beliefs, attitude those are safe, supportive and healthy (Hornor, 2017). These approaches strengthen resiliency at population level to reduce adverse childhood experiences (Hornor, 2017; Arafat, 2017). Legislations aiming at reducing the social disparities such as wage, affordable education, easy access to mental health care system are the example of resiliency promotion at population level (Arafat et al., 2018; Horner, 2017; Arafat, 2017). Promoting resiliency optimally should cover all the extents, that is, at society level, community level, family level and certainly individual level (Horner, 2017). Multidisciplinary approach involving government, nongovernment, national, international, intersectoral harmonious approach is necessary (Arafat, 2017; Hornor, 2017). Child maltreatment, particularly sexual abuses, should be controlled by the central policy approach (Hornor, 2017). On the contrary, strategies focusing on the communication skills, healthy peer relationship, involvement of family, school, parents should be practiced as microlevel initiatives (Hornor, 2017). Few examples of microlevel activities can be mentioned, such as parenting training, prevention of bullying, drug misuse awareness (Hornor, 2017). Few examples of community involvement 
in building resiliency include control of violence, shelters for homeless population, domestic violence awareness build up, and early identification of risky individuals (Hornor, 2017). Working with temperament of individual child, identification of strength and weakness of individual child, physical exercise, extra-curricular activity involvement, promoting healthy peer relationship could be a few examples of individual strategies (Horner, 2017). Relaxation exercise may be beneficial to increase resiliency for children as well as adults to cope up with stressors (Franke, 2014). A specialized services team, headed by a paediatrician can play an important role in identifying the risk factors, reducing the effects of vulnerable factors along with raising the resiliency factors in persons both individually and within families (Franke, 2014). Health promotional activities, strategies and researches would certainly need to foster the resiliency as well as to decrease the effects of vulnerability among children (Arafat, 2017; Moreno et al., 2016). Schools can play vital roles to identify and report vulnerability issues towards parents as well as other social agencies to address it properly (Arafat et al., 2018).

\section{Asset-Focused Intervention Strategy}

Provision of necessary assets counterbalance the effects of poverty. Thus, this strategy focusses on making available elements those are necessary for normal development in adequate amount (Masten \& Gewirtz, 2006). However, it can be questionable to implement in developing countries where such resources are limited along with such political commitment.

\section{Risk-Focused Intervention Strategy}

Working on the risk factor highlights the risk focused intervention. Reduction of overall risk, modify the risk to less disastrous and/or elimination of risk factors are targeted by this strategy (Masten \& Gewirtz, 2006). Identification of risk factors as well as identification of risky individual, both can be effective based on the available resources. Health promotion interventions highlighting the risk factor and/or risk individual may play the central role (Masten \& Gewirtz, 2006; Arafat, 2017). For instances few strategies have been mentioned here such as removal of heavy metal from environments, adequate sleep on back, smoking reduction in pregnancy, firearm control legislation, adequate nutrition during prenatal period and so on (Masten \& Gewirtz, 2006).

\section{Protection-Focused Intervention Strategy}

Protection focused strategies highlight protection from our aversive life events or stressors (Masten \& Gewirtz, 2006).
For instance, car seats for infant, crisis nurseries, foster care, sheltering for the domestic violence, telephone services and other safety planning for child(ren) related to violence, abduction, or sexual abuse have shown effective as the protection focus intervention strategies. Specially tailored parenting program named as Parenting Through Change program is highlighted among the aforementioned strategies (Masten \& Gewirtz, 2006).

\section{Multi-Focused Strategy}

Sorting out a perfect strategy usually may be illusive in reality. Amalgamation of strategies can be an important step to consider to promote resilience of an individual initially and a society later (Masten \& Gewirtz, 2006). Customizing the strategies based on the individual's personality construct, available resources and limitation should consider, as every individual is unique in some extent (Masten \& Gewirtz, 2006). Harmonization between strategies, between person and society, family, school, community, social policy, political commitments is a vital issue to promote resiliency (Masten \& Gewirtz, 2006).

\section{CONTROVERSIES IN VULNERABILITY AND RESILIENCY}

All persons facing the same stressors and/or challenges, however, responding differently. Few are growing as resilient and others as vulnerable (Hornor, 2017). Difficulties in the prediction of vulnerability and resiliency along with nurturing children with guarantee of resiliency is really a challenge. Moreover, exploration of childhood of great personalities revealed huge stressful events in their early childhood. Same environment can direct differently for different individual, for same individual in different time (Broekman, 2011; Hornor, 2017). Preloaded genetic and biological components along with personal experiences based on emotional interaction of attachment and social learning influences the resilience (Broekman, 2011). Personal and cultural factors play a dynamic role on developing resiliency (Broekman, 2011).

\section{CONCLUSION}

Resiliency and vulnerability are the integral characteristics of human being, which is fairly difficult to explain as well as to predict. Though, the precise mechanisms of resiliency and vulnerability are yet to be explained, it has been relatively established that active coping is associated with the development of resiliency and passive coping is associated with vulnerability. Initially, it was thought as a person-centred matter; however, later it revealed as complex interactions between an individual 
and his/her surrounding ecology. Enduring pattern of individual characteristics comprised of good intellectuality, adequate emotion regulation, good attachment in early childhood, positive self-esteem, optimistic and altruistic attitude, an excellent capability of learned helpfulness and active coping strategy are thought to be beneficial for resiliency. Experiences of early period of childhood play a pivotal role in the evolution of brain and the development of resiliency or vulnerability. Increased sensitivity to stress, inadequate social and psychological resource, inadequate social support, developmental delays, abusive parenting, and maladaptive behaviours were found as vulnerable factors. Building resiliency demands is a combination of macro- and microlevel initiatives, where microlevel strategies focus on community, family and individual stage and macrolevel strategies formulate the principles such as economic policy, social dogma, community set up and cultural beliefs. Multidisciplinary approach involving government, non-government, national and international bodies is necessary.

\section{REFERENCES}

Anderson NB. Levels of Analysis in Health Science: A Framework for Integrating Sociobehavioral and Biomedical Research. Annals of the New York Academy of Sciences, 1998;840:563-576.

Arafat SMY. Mental Health Promotion or Public Mental Health: The Time Demanded Area. J Behav Heal. 2017;6(1):1-3.

Arafat SMY, Majumder MAA, Kabir R, Papadopoulos K, Uddin MS. (2018). Health Literacy in School. In V. E. Papalois \& M. Theodosopoulou (Eds.), Optimizing Health Literacy for Improved Clinical Practices 2018;(pp. 175-197). Hershey, PA: Medical Information Science Reference.

Beutel ME, Tibubos AN, Klein EM, Schmutzer G, Reiner I, Kocalevent R.-D, Brähler, E. Childhood adversities and distress - The role of resilience in a representative sample. PLOS ONE. 2017;12(3):e0173826.

Broekman BFP. Stress, vulnerability and resilience, a developmental approach. Eur J Psychotraumatol. 2011;2(1):7229.

Carlson NR. Physiology of Behavior (11th ed.). Boston: Pearson. 2013;602-606. ISBN 978-0-205-23939-9.

Charney DS. The psychobiology of resilience and vulnerability to anxiety disorders: implications for prevention and treatment. Dialogues Clin Neurosci. 2003;5(3):207-221.

\section{ACKNOWLEDGEMENTS}

None

\section{CONFLICT OF INTEREST}

None

\section{DECLARATION OF ETHICS}

The study was conducted complying the declaration of Helsinki 1964. No formal permission was needed to conduct the review.

\section{DECLARATION OF INFORMED CONSENT}

Not applicable

\section{FUNDING}

Self-funded

Chandler GE, Roberts SJ, Chiodo L. Resilience intervention for young adults with adverse childhood experiences. J Am Psychiatr Nurses Assoc. 2015;21(6):406-416.

Chetty S, Friedman AR, Taravosh-Lahn K, Kirby ED, Mirescu C, Guo $F$, et al. Stress and glucocorticoids promote oligodendrogenesis in the adult hippocampus. Mol Psychiatry 2014;19:1275-1283.

Compas EC, Jaser SS, Dunn MJ, Rodrguez EM. Coping with Chronic Illness in Childhood and Adolescence. Annu Rev Clin Psychol. 2012;8:455-480.

Daniel B. Concepts of adversity, risk, vulnerability and resilience: a discussion in the context of the 'child protection system'. Social Policy and Society, 2010; 9(2):231-241.

Evans GW, Kim P. Childhood poverty, chronic stress, selfregulation, and coping. Child Dev Perspect. 2013;7(1):43-48.

Fink G. Stress: definition and history; in Squire LR (ed): Encyclopedia of Neuroscience. Oxford, Academic Press, 2009;9:549-555.

Franke H. Toxic Stress: Effects, Prevention and Treatment. Children, 2014;1(3):390-402.

Franklin TB, Saab BJ, Mansuy IM. Neural Mechanisms of Stress Resilience and Vulnerability. Neuron. 2012;75(5):747-761. 
Gershoff E, Aber J, Raver C. Child poverty in the United States: An Evidence-based conceptual framework for programmes and policies. In R. Lemer, D. Wertlieb \& F. Jacobs (Eds). Handbook of applied developmental science. 2003;4:1-136. California:Sage Publications Inc.

Hammen C, Kim EY, Eberhart NK, Brennan PA. Chronic and acute stress and the prediction of major depression in women. Depress Anxiety. 2009;26(8):718-723.

Hornor G. Resilience. J Pediatr Health Care. 2017;31(3):384-390.

Janse AJ, Uiterwaal CSPM, Genke RJBJ, Kimpen JLL, Sinnema G. A difference in perception of quality of life in chronically ill children was found between parents and pediatricians. J Clin Epidemiol. 2005;58:495-502.

Judge S. Resilient and Vulnerable At-Risk Children. Journal of Children and Poverty. 2005;11(2):149-168.

Knapp PK, Harris ES. Consultation-liaison in child psychiatry: a review of the past 10 years. Part I: clinical findings. J Am Acad Child Adolesc Psychiatry. 1998;37:17-25.

Masten AS, Gewirtz AH. Vulnerability and resilience in early child development. In Blackwell handbook of early childhood development 2006;22-43.

Masten AS, Barnes AJ. Resilience in Children: Developmental Perspectives. Children (Basel). 2018;5(7): 98.

Miller LH, Smith AD. The stress solution: An action plan to manage the stress in your life. New York: Pocket Books. 1993.

Moreno C, García-Moya I, Rivera F, Ramos P. Characterization of Vulnerable and Resilient Spanish Adolescents in Their Developmental Contexts. Front Psychol. 2016;7:983.

Romer G. Assessing stress in children: A literature review. Presentation at the 1993 Mid-South Educational Research Association Conference, 1993;1-17. New Orleans, LA.

Selye, H. (1976). Stress in Health and Disease. Stoneham, MA Butterworth.

Selye $\mathrm{H}$. The Story of the adaptation syndrome. Montreal:ACTA, Inc., Medical Publisher. 1952.
Stuber ML, Schneider S, Kassam-Adams N, Kazak AE, Saxe G. The medical traumatic stress toolkit. CNS Spectrums. 2006;11:137142.

Szabo S, Tache Y, Somogyi A. The legacy of Hans Selye and the origins of stress research: A retrospective 75 years after his landmark brief "Letter" to the Editor of Nature. Stress. 2012;15:472-478.

Terzian M, Moore KA, Nguyen HN. Assessing Stress in Children and Youth: A Guide for Out-of-school Time Program Practitioners. 2010. www.childtrends.org/wp-content/uploads/2010/10/Child_ Trends-2010_10_05_RB_AssessingStress.pdf lassessed 13 Aug 2018).

Thoresen CE, Eagleston JR. Chronic stress in children and adolescents. Theory Pract. 1983;22:48-56.

Van Rensburg A, Theron L, Rothmann S. A review of quantitative studies of South African youth resilience: Some gaps. S Afr J Sci. $2015 ; 111(7 / 8): 9$.

Wood SK, Bhatnagar, S. Resilience to the effects of social stress: Evidence from clinical and preclinical studies on the role of coping strategies. Neurobiol Stress. 2015;1:164-173. 
